\title{
Design of block-copolymer-based micelles for active and passive targeting
}

\author{
Jérôme G. J. L. Lebouille, ${ }^{1}$ Frans A. M. Leermakers, ${ }^{2}$ Martien A. Cohen Stuart, ${ }^{2,3}$ and Remco Tuinier ${ }^{4,5, *}$ \\ ${ }^{1}$ DSM Biomedical, P.O. Box 18, 6160 MD Geleen, the Netherlands \\ ${ }^{2}$ Physical Chemistry and Soft Matter, Wageningen University, Stippeneng 4, 7608 WE Wageningen, the Netherlands \\ ${ }^{3}$ Physics of Complex Fluids, Department of Science and Technology, University of Twente, P.O. Box 217, 7500 AE Enschede, the Netherlands \\ ${ }^{4}$ Laboratory of Physical Chemistry, Department of Chemical Engineering and Chemistry \& Institute for Complex Molecular Systems, \\ Eindhoven University of Technology, P.O. Box 513, 5600 MB Eindhoven, the Netherlands \\ ${ }^{5}$ Van 't Hoff Laboratory for Physical and Colloid Chemistry, Debye Institute \& Debye Institute, Padualaan 8, 3584 CH, \\ Utrecht University, the Netherlands
}

(Received 29 March 2016; published 21 October 2016)

\begin{abstract}
A self-consistent field study is presented on the design of active and passive targeting block-copolymeric micelles. These micelles form in water by self-assembly of triblock copolymers with a hydrophilic middle block and two hydrophobic outer blocks. A minority amount of diblock copolymers with the same chemistry is taken to coassemble into these micelles. At the end of the hydrophilic block of the diblock copolymers, a targeting moiety (TM) is present. Assuming that the rotation of the micelle towards the target is sufficiently fast, we can elaborate a single gradient cell model, wherein the micelle is in the center and the receptor (R) substrate exists on the outer plane of the spherical coordinate system. The distribution function of the targeting moiety corresponds to a Landau free energy with local minima and corresponding maxima. The lowest minimum, which is the ground state, shifts from within the micelle to the adsorbing state upon bringing the substrate closer to the micelle, implying a jumplike translocation of the targeting moiety. Equally deep minima represent the binodal of the phase transition, which is, due to the finite chain length, first-order like. The maximum in-between the two relevant minima implies that there is an activation barrier for the targeting moiety to reach the receptor surface. We localize the parameter space wherein the targeting moiety is (when the micelle is far from the target) preferably hidden in the stealthy hydrophilic corona of the micelle, which is desirable to avoid undesired immune responses, and still can jump out of the corona to reach the target quick enough, that is, when the barrier height is sufficiently low. The latter requirement may be identified by a spinodal condition. We found that such hidden TMs can still establish a TM-R contact at distances up to twice the corona size. The translocation transition will work best when the affinity of the TM for the core is avoided and when hydrophilic TMs are selected.
\end{abstract}

DOI: 10.1103/PhysRevE.94.042503

\section{INTRODUCTION}

Nanoparticles are nowadays used for pharmaceutical applications [1-4]. The drug delivery performance of such particles depends on control over particle size, drug loading, and active targeting capability. One can use nanosized micellar structures formed by a combination of (different) amphiphilic molecules in a selective solvent. Nanoprecipitation enables the formation of self-assembled block copolymer spherical micelles, even for rather hydrophobic copolymers. Potent drugs are often rather hydrophobic ingredients for which polymeric micelles are promising carriers, with potential for controlled encapsulation of compounds at high loadings. Additionally, using polymeric micelles offers routes to control release, stability, and biodistribution of active agents in the body for both passive and active targeting [5-13]. Here, we focus on water as the solvent and consider block copolymers with two apolar and one polar block, the so-called ABA block copolymers which readily form spherical flowerlike micelles. To assure active targeting, we propose to combine the triblock copolymers with $\mathrm{AB}$ diblocks with targeting moieties at the end of the hydrophilic block B. Within the spherical micelles, the hydrophobic entities are collected in a compact core, whereas the water-soluble blocks remain hydrated and

\footnotetext{
*r.tuinier@tue.nl
}

form a corona. The diblocks are designed in such a way that their B blocks are preferably located outside the hydrophobic core of the micelle. As hydrophobic blocks A we envision poly-(lactic-coglycolic) acid (PLGA) and as hydrophilic block B polyethylene oxide (PEO). Although PEO is nondegradable, it is nontoxic and can easily be removed from the body by the normal excretion (renal) pathways as long as the molar mass is below $20 \mathrm{kDa}$ [14]. The hydrophobic part consists of PLGA, an FDA approved and well known biodegradable polymer used in commercially available drug delivery applications $[4,15]$. In principle, PEO and/or PLGA can be replaced by other hydrophilic and hydrophobic (co)polymers.

The goal is to obtain more fundamental insight in the behavior of copolymers with targeting moieties within composite micelles. The method employed, the Scheutjens-Fleer self-consistent field (SF-SCF) theory [16-18], successfully used earlier to theoretically study the triblock copolymer micelle structure and stability $[19,20]$. It was found that by implementing a model wherein segments are represented as amorphous beads, i.e., $\mathrm{A}_{60} \mathrm{~B}_{60} \mathrm{~A}_{60}\left(\mathrm{PLGA}_{60} \mathrm{PEO}_{60} \mathrm{PLGA}_{60}\right.$; 7.5-6.0-7.5 kDa), there exists a set of interaction parameters that leads to structural properties of micelles that compare favorably with the ones found experimentally using the solvent precipitation method [21]. Once such micelles have formed, we envision that the core becomes unresponsive, i.e., glassy, and this allows us to focus here exclusively on what happens in the molecularly quenched, but conformationally adaptive 
corona. Basically, we take the core to be a solid particle (composed of units of type A; PLGA) of which the size is set equal to that found for the micelle cores. We graft both ends of a hydrophilic chain $\mathrm{B}_{60}(\mathrm{PEO} ; 6.0 \mathrm{kDa})$ onto the core so that a spherical brush is formed of looped chains. The grafting density of this brush is set equal to the aggregation number found for the self-assembled micelles. Next, we consider that onto the same core there is one chain grafted by one end only, the AB (PLGA-PEO) diblocks leaving one free dangling chain end. We take the chemistry of this chain identical to that of the triblocks, only the length of the B (PEO) block of this minority chain is a free variable. The key is that the free end is given some different properties, consistent with the idea that this end is functionalized by a targeting moiety (TM). The focus of this paper is to understand the behavior of the minority chain and in particular understand the whereabouts of the functional free end. In more detail, our attention will be on the translocation transition of the minority chain end going jumplike from being inside the corona to outside the brush, a transition which is driven by the potential energy gain received when the TM is in contact with a receptor $(\mathrm{R})$. In our simple cell model, the receptor is expected to be present homogeneously along the surface that surrounds the central micelle.

Biodistribution of micelles in the human body is mainly dependent on their size, surface, and corona structure. Micellar size control exploits passive targeting via the enhanced permeation and retention (EPR) effect [6,22-25]. For targeting purposes we focus on tumor cells and tissue as an example. Improving chemotherapy offers a major challenge in human health treatments. Of course, other diseased tissues will demand different targeting moiety (TM) receptor (R) combinations. Especially for micellar accumulation in tumor tissues the size and surface structure is of crucial importance for passive targeting.

For active targeting, one needs to incorporate moieties for specific targeting into or onto the micellar corona. Ideally targeting moieties are included that are specific for the targeted tissue. When the connection between targeting moiety and receptor is strong enough, active targeting will be enabled. From the biological perspective, there are a number of requirements. First of all, the micelle needs to have the proper chemistry. It is known that polyethylene oxide micelles are stealthy (noninterfering with the immune system) and have long blood circulation times [26]. Hence, we take the interaction parameter for the B group with water accordingly. To avoid any undesired interaction with the immune system, we believe that it is favorable to have the chain end well inside the micelle corona, that is under normal operation conditions, when the micelle is far from its target. This requirement can be implemented in two ways. In the first option, we take the length of the minority chain to be less than half the length of the loops that form the bulk of the corona, i.e., $N_{\mathrm{B}}<30$. To go outside the corona will imply extra stretching of the minority chain which is entropically unfavorable. The second option is to give the free chain end an affinity for the core of the micelle. Typically, this already occurs when the targeting moiety is hydrophobic. Then, there is a natural tendency that the TM is associated to the core. In the adsorbed state of the TM to the cote the TM is obviously inside the corona, even when the length of the minority chain exceeds that of the half-loop length. Experimentally, when the TM is hydrophobic, it may be locked inside the core and is subsequently unable to escape from it. Here we will ignore this scenario because we take the core to be composed of A segments only (the core is a boundary condition in the current calculations). On top of the hydrophobic interaction we may introduce some extra surface affinity, e.g., due to some specific interactions. Of course, this will complicate our analysis because there will be a free energy barrier associated with the detachment of the end group from the core and the time limiting step for the translocation transition may well be associated with the desorption process, rather than being associated to the entropic stretching towards the adsorbing surface itself.

The design, manufacturing, and characterization of ABA block copolymer micelles composed of rather hydrophobic copolymers was reported previously [19-21]. It was demonstrated that tuning the size and loading can be achieved by using triblock copolymers with a particular chain length combined with active ingredients. The synthesis and incorporation of targeting moieties onto existing particles is difficult and tedious. In most cases, the targeting function on a nanoparticle or micelle is established after its formation. Since most of these processes demand surface chemistry instead of physical interactions, a purification step is mandative to remove reactants [27-31]. To omit the post processing and purification steps, we propose a single micellar processing method. This allows incorporation of the targeting moieties, present on the diblocks, from the start of the micellar production process using the nanoprecipitation method. It is noted our approach and the results can also be applied towards targeting applications using polymer coated nanoparticles.

We first give elementary background information on the modeling method, focusing on the main approximations. We then provide detailed information on the model used and discuss the relevant parameters. We present the structure of the polymeric micelle that is used to further study the escape or translocation transition of the minority chain. In somewhat more detail, we will subsequently discuss how we are going to analyze the translocation transition of the TMs. More specifically, we will elaborate on the Landau free energy as a function of the position of the free end. This free energy will be used to judge when the jumplike phase transition occurs and what the origin is of the free energy barriers that are encountered. We will elaborate on a typical example in the limit of an excess of receptors available. The main results are collected in diagrams of state wherein the binodal and spinodal curves are collected. These diagrams facilitate the search for the most optimal properties of the minority chain in a composite micelle. In our discussion and conclusions, we sum up our theoretical modeling results with regard to the design of an active targeting capacity for composite micelles.

\section{THEORY}

This section contains two major parts. First focus is on outlining the SF-SCF theory. We explain the main approximations of the modeling in combination with the model that is used for the copolymer self-assembly. For more detailed information we refer to $[19,20]$. Subsequently, the micelle is presented that is selected as a representative case in some 
detail and reproduces a result from a previous study [20] for ease of reference. Next, we elaborate on the model used for the remainder of this paper. The cores of the micelles are molecularly quenched, implying that the aggregation numbers and core structure are frozen in. Important is that the micelles remain responsive in the micelle corona for which the chains can conformationally rearrange. In the second section, we elaborate on the use of the Landau free energy (see [32]) by elaborating on a specific example, and explain in detail the physics that is accounted for. In this section, attention is being paid to definitions of the binodal and spinodal from the SF-SCF results. Finally, the relevant SF-SCF parameters are discussed which will be investigated on their influence on TM-R contact.

\section{A. SF-SCF theory}

Making use of the mean field approximation, and more specifically the Bragg-Williams approximation (more about this below), allows to formulate for a system a free energy in terms of two conjugate profiles, namely, the measurable volume fraction (dimensionless concentrations) $\varphi(r)$ and complementary segment potential $u(r)$ distributions. In a way these potentials are external potentials because we use them to obtain statistical weights, the so-called Boltzmann weights as in a barometric height equation $G(r)=\exp [-u(r)]$. Here and below, all energy quantities are made dimensionless by normalizing them with the thermal energy $k_{B} T$. The weights are not fixed, but the segment potentials appear to be a function of the volume fractions. The functionality follows uniquely from the free energy optimization. As a result, the segment potential takes the interaction of a given segment with its surroundings into account. These interactions are parametrized by Flory-Huggins interaction parameters $\chi_{X-Y}$ wherein both $X$ and $Y$ are segment (or solvent) types. A positive value means that the $X Y$ contacts are less favorable than the average of $X X$ and $Y Y$ contacts. Hence, a sufficiently large positive value implies segregation. Low values or negative values are needed for proper miscibility.

Interestingly, not only do the potentials depend on the volume fractions, the inverse is also true: the volume fractions are a function of the segment potentials. To implement this dependence, it is necessary to invoke a chain model. Here, the freely jointed chain model is used, which guarantees that neighboring segments of the chain are at a distance of a bond length. However, as no further through-bond correlations are imposed, a chain can also fold back on previously occupied places. Let us next focus on a particular conformation $c$ of the chain, completely specified by the coordinates of the individual segments. This conformation is found to have a particular potential energy $u_{c}$, which is computed by the sum over the segment potentials felt by the consecutive segments along the contour of conformation $c$. The statistical weight of this conformation is then again given by a Boltzmann weight $G_{c}=\exp \left(-u_{c}\right)$. Such weight is found for all possible and allowed conformations. With these statistical weights one can construct the overall volume fraction distribution. Of course, this requires a sum over the contributions of all possible and allowed conformations. As the total number of possible conformations is very large indeed, these sums suggest an insurmountably large job. Fortunately, there exists an elegant propagator method that copes with it efficiently, in a computation time which is linearly proportional to the number of segments in the chain (not shown).

Summarizing, characteristic for the SCF method we have

$$
\varphi[\{u\}] \leftrightarrow u[\{\varphi\}] .
$$

In words, the left hand side of this equation says that the volume fractions can be computed from the potentials and the right hand side says the opposite. In the case that the set of potentials and volume fractions is consistent, that is, that the potentials both follow from and determine the same volume fractions and vice versa, we refer to the solution being self-consistent. There is a pitfall, namely, as specified thus for there are many SCF solutions to the equations. That is why one additional requirement is necessary to make the solution unique. This additional requirement specifies the use of an (in)compressibility relation. Here, we opt the system to be incompressible and therefore we impose

$$
\sum_{X} \varphi_{X}(r)=1
$$

for each coordinate $r$.

In very few cases it is possible to solve the self-consistent field solution analytically. It is of no surprise that the selfassembly of copolymers into spherical micelles is not one of these rare cases. As a result, we have to implement the equations into a computer model and solve for the fixed point numerically. Invariably, one needs to discretize the equations somehow. Here, we follow the SCF method of Scheutjens and Fleer (SF-SCF) and make use of lattice of sites. The characteristic length of a lattice site is taken equal to the bond length, so that one polymer segment exactly fits into a lattice site. Solvent molecules are taken to have the same volume as a segment for simplicity.

For many problems, one can recognize some sort of symmetry element. For example, in spherical micelles we can easily imagine that the relevant gradients in polymer densities occur in the radial direction and that the density fluctuations in a shell of lattice sites with equal distance to the center are very small. This motivates us to reduce the three-dimensional system and implement this in a one-gradient spherical geometry. Then, lattice layers are numbered starting from the center $r=1,2, \ldots, M$. Naturally, the number of lattice sites $L(r)$ in layer $r$ scales as $L(r) \propto r^{2}$. By ignoring the density fluctuations in the layer (mean field approximation) we can implement the volume fraction $\varphi_{X}(r)=n_{X}(r) / L(r)$, wherein $n_{X}(r)$ is the number of segments of component $X$ in layer $r$. At the upper boundary at $r=M$ typically reflecting boundary conditions apply. This is implemented by forcing all density gradients to be zero at this boundary, i.e., $\varphi_{X}(M+1)=$ $\varphi_{X}(M)$. Usually, the value of $M$ is taken very large, far from the micelle, which is positioned at the center of the spherical coordinate system, where the density gradients have vanished (bulk). Then, these reflecting boundary conditions are inconsequential for any physical property of the system.

Below, we consider a spherical "micelle" in the vicinity of a surface. This typically cannot be captured in a spherical geometry. Instead, one might be tempted to use a two-gradient cylindrical coordinate system. Below it is, however, shown that the problem of interest can be studied using a single 
gradient spherical geometry. More specifically, we are going to assume that the rotational diffusion of the micelle is quick enough so that the probe will find the receptor even when some rotation of the micelle is needed. Then, having a surface in all directions around the micelle (as is explicitly implemented) is a reasonable approximation. This idea is realized by imposing an impenetrable boundary at layer $r=M+1$, that is, at layer $r=M+1$ the volume fraction of a segment with type $R$ is unity. Segments with type $X$ feel this boundary when they sit in layer $z=M$ through the interaction parameter $\chi_{X-R}$. Also, the solvent $W$ has an interaction with this surface $\chi_{W-R}$ and typically segments may adsorb onto the surface only when a solvent molecule is displaced from it, hence, micelle adsorption is expected only when $\Delta \chi_{R} \equiv \chi_{X-R}-\chi_{W-R}<0$, otherwise the solvent is preferentially adsorbed at the surface. It is well known that polymer chains experience conformational entropy losses when they are directly next to an impenetrable wall; the reason for this is that the orientation of bonds is hindered as these cannot enter into the solid phase. To compensate for this, it is necessary that $\Delta \chi_{R}<\chi_{\text {critical }}$, for the segment $X$ (part of a chain) to adsorb, where in good approximation $\chi_{\text {critical }}=-1$ for typical segments in a polymer chain. The critical adsorption energy for end segments is expected to be less negative as these segments have just one bond connected to them. The critical adsorption is also a function of the solvent quality. To understand this, one should realize that a segment next to the surface cannot interact with the solvent in all directions. Hence, it can avoid one contact with the solvent and thus there is an increased tendency to be next to the (receptor) surface. Below the affinity of the probe for the (receptor) surface is implemented using the parameter $\chi_{\mathrm{TM}-\mathrm{R}}$. In our approach, it is assumed that a large excess of receptors is available. Several authors have studied the effect of the availability of receptors and we refer to their papers for interested readers [33-36].

In the SF-SCF method, the molecules are discretized, they are taken to be composed of segments numbered

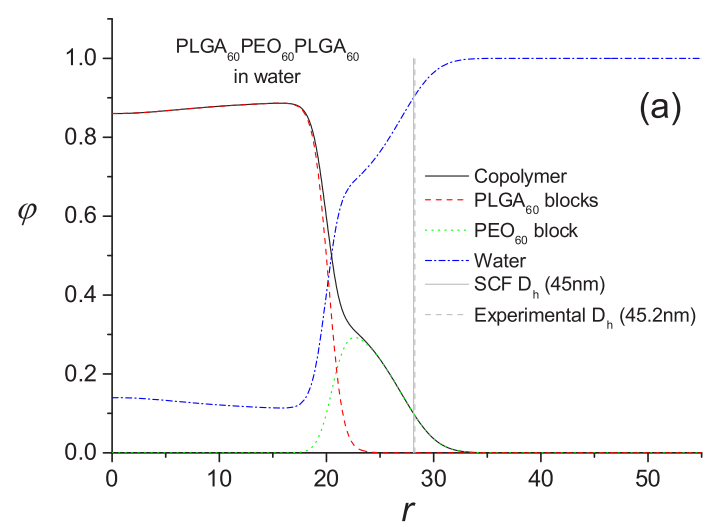

$s=1,2, \ldots, N$. We refer to $N$ as the chain length, but possibly the degree of polymerization is a better term for it. We do not take any structural features on the segment level into account. However, we do allow for copolymer type of interactions, e.g., one segment is hydrophobic and the other is hydrophilic. We do this by specifying the Flory-Huggins interaction parameters for each segment type accordingly (see Sec. II C).

\section{Molecular model and self-assembly}

The study of the self-assembly of copolymers into, e.g., thermodynamically equilibrated spherical micelles is a key application of the Scheutjens-Fleer self-consistent field (SFSCF) theory. In previous studies $[19,20]$ we considered the formation of spherical micelles composed of $\mathrm{A}_{60} \mathrm{~B}_{60} \mathrm{~A}_{60}$ where $\mathrm{A}_{60}(7.5 \mathrm{kDa})$ is the hydrophobic block mimicking PLGA and $\mathrm{B}_{60}(6.0 \mathrm{kDa})$ the hydrophilic block representing PEO (also called PEG, polyethyleneglycol).

Let us now formulate a key property of the molecular model. The set of parameters should result in micelles wherefore the core and corona sizes are of the same order of magnitude. Even though the topological stability requires the size of the core to be less than that of the corona, as explained, the dynamic process by which the micelles form is more likely characterized by the situation that the micelle corona is somewhat smaller than that of the core. We have opted for a driving force for micellization using a modest value $\chi_{\text {LGA- } \mathrm{H}_{2} \mathrm{O}}=1.6$, whereas the solvent quality for PEO is marginal: $\chi_{\mathrm{EO}-\mathrm{H}_{2} \mathrm{O}}=0.4$. This value ensures that the corona is well solvated and the pressure in the brush provides the stopping mechanism for the self-assembly of the micelles. The spatial segregation of A segments in the core and the $\mathrm{B}$ segments in the corona is improved by a repulsion between these segments. Here, we have used $\chi_{\text {LGA-EO }}=1.0$ for simplicity. The justification of this set that it largely obeys with our requirements. We stress that small modifications of the parameter set do not perceptibly affect the results.

In Fig. 1(a) the micelle structure, wherein the radial volume fraction profiles for the polar segments, the apolar segments

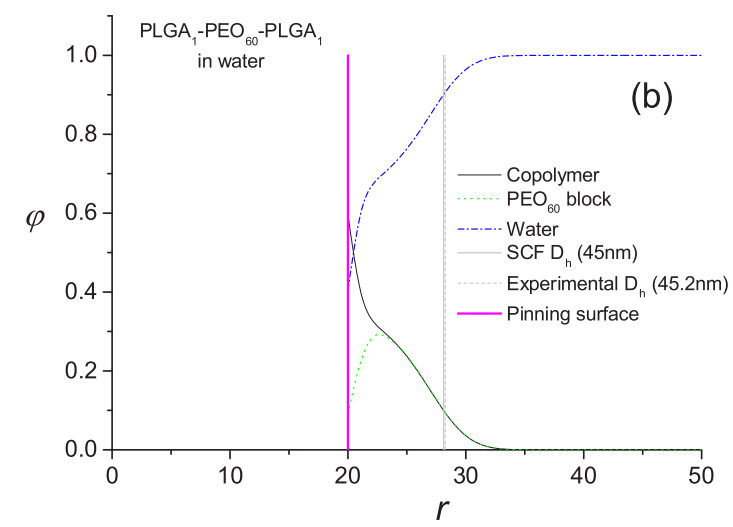

FIG. 1. Equilibrium radial density profiles. (a) The equilibrium radial density profile of $\mathrm{PLGA}_{60} \mathrm{PEO}_{60} \mathrm{PLGA}_{60}$ in a spherical coordinate system. The profiles for the apolar segments PLGA, the polar segments PEO, and the solvent $W$ (water) are given. Parameters $\chi_{\text {LGA- } \mathrm{H}_{2} \mathrm{O}}=1.6$, $\chi_{\text {LGA-EO }}=1.0$, and $\chi_{\mathrm{EO}-\mathrm{H}_{2} \mathrm{O}}=0.4$. The aggregation number $g=237$. The grand potential $\Omega=10 k_{B} T$ [19]. (b) The equilibrium radial density profile of the brush made of $\mathrm{PLGA}_{1} \mathrm{PEO}_{60} \mathrm{PLGA}_{1}$ where the A units are pinned to be next to the particle surface, with radius $R=20$ (lattice units). The particle is made of segments of type A. In line with the aggregation number of the micelle of panel (a), the grafting density $\sigma=0.047$. Here, the radial coordinate is given in lattice sites units. For the radial coordinate, we have used a lattice site dimension (which corresponds to $0.8 \mathrm{~nm}$ ). 
and the solvent molecules are given, here it is observed that the core has approximately a homogeneous density of polymer. The small decrease of density towards the center of the core is attributed to an inhomogeneous stretching of the corona chains, being largest near the core-corona interface (where they then pack a bit more efficient). The solvent in the core is not extremely low, which is consistent with the relatively low value for the $\chi_{\mathrm{LGA}-\mathrm{H}_{2} \mathrm{O}}$ that was adopted to mimic the assembly at the quench conditions. The corona has a much lower density, as it is well solvated. The profile is quasiparabolic, and the height (dimension) of this brush is expected to scale linearly with the length of the B block.

Inspection reveals that the core radius, which in this case is approximately 20 lattice units, is slightly larger than that of the corona. The latter may be estimated from the hydrodynamic diameter of the micelle which is found to be 56 lattice units $(45 \mathrm{~nm})$. This is consistent with one of the targets that were used for the molecular model. The overall dimension of the micelle is in good agreement with the micelles found in the experimental condition. From the modeling point of view, the micelles are stable. For example, we have selected a micelle with a grand potential (work of micelle formation) $\Omega=10 k_{B} T$. This value is thought to be compensated by translational entropy of the micelle, and thus represents the situation that the micelles are far apart (dilute regime). Thermodynamic stability requires that the slope $\partial \Omega / \partial g$ is negative, which was shown to be the case in a previous study $[19,20]$. The aggregation number, that is, the number of triblock copolymers in the micelle, is predicted to be $g=237$. Again, the order of magnitude is consistent with experimental data. We refer that a brush of loops with chains of length $N_{\mathrm{B}}=60$ is usually seen as a brush composed of tails of length $N_{\mathrm{B}}=30$ with double grafting density. We have checked that this is a good approximation also in this case (result not shown).

In the following, we will use the micelle presented above and analyze the behavior of a minority chain that is added to this micelle. The distortion of the entire micelle by the minority chain is accounted for explicitly. The analysis is facilitated when the micelle is replaced by a simpler structure. Considering the sharp interface between the core and the corona, we argue that it is possible to replace the core by a solid particle and replace the corona by a set of polymers that are anchored by both ends to this solid particle. In Fig. 1(b), we present the structure of a brush of loops connected to a spherical particle. The parameters are chosen such as to resemble the corona of the micelle presented in Fig. 1, that is, the number of grafted "loops" is equal to the aggregation number $g$, and the solvent quality is again set to $\chi_{\mathrm{EO}-\mathrm{H}_{2} \mathrm{O}}=0.4$. The differences in radial structure of Figs. 1(a) and 1(b) are minor which proves that the model of Fig. 1(b) is sufficiently accurate to mimic a triblock copolymer micelle for our purpose as used below. The most significant differences between the two radial profiles occur just next to the "core"-corona interface. As explained above, the entropic penalty for chains next to a sharp interface results in a small depletion of polymers and a small adsorption of solvent. In the micelle, the core-corona interface is less sharp and then the latter effects do not occur. One can easily correct for this entropy loss by adding an adsorption affinity of the corona chains for the core. This is not implemented here.

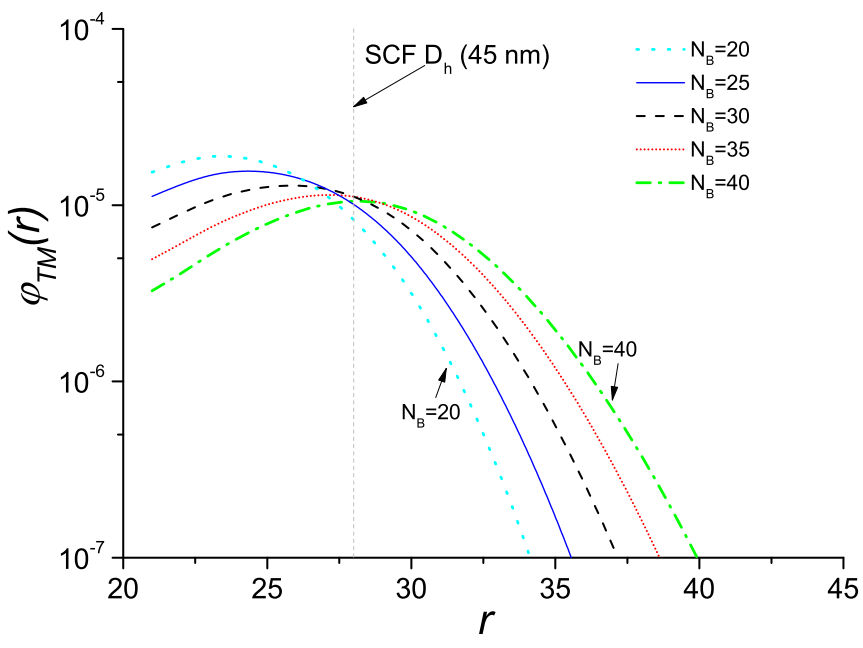

FIG. 2. The equilibrium radial density profile of the end point (TM) of the minority chain in the corona of the micellelike particle given by Fig. 1(b) in log-lin coordinates and lattice site units. The minority chain length is modified from $N_{\mathrm{B}}=20$ to 40 as indicated. The curve for the chain length 30 is dashed. The dashed gray line represents the hydrodynamic diameter $\left(D_{h}\right)$.

\section{A minority chain within the corona of the micelle}

Following the line of arguments, we take the corona structure presented in Fig. 1(b) and admix in this structure one minority chain with a structure $\mathrm{A}_{1} \mathrm{~B}_{N_{\mathrm{B}}} \mathrm{TM}_{1}$. It was verified that a few minority chains do not strongly influence the colloidal stability of the micelle. The focus here is on the radial profile of a single minority chain inside the corona made of $A_{1} B_{60} A_{1}$. More specifically, our interest is in the profile of the end segment referred to as TM. In Fig. 2, we present a number of radial volume fraction profiles of the TM segment in semilogarithmic coordinates. One of the profiles, that is for $N_{\mathrm{B}}=30$, is dotted. This case represents a mimic of the majority chains that make up the bulk of the corona. When the minority chain is shorter, the chain is more likely to fully remain inside the corona. Longer chains typically escape from the corona. The shape of the free end distribution is not much affected by the length and thus not much affected whether or not it is predominantly inside or outside the corona; the end points are distributed throughout the corona, but the most likely position is further from the core when the probe length is larger. The average position of the end point, e.g., measured by the first moment over the end-point distribution, grows approximately linear with length $N_{\mathrm{B}}$ and in this dependence there is no discontinuity around $N_{\mathrm{B}}=30$.

For the remainder of the discussion it is important to mention that upon the transition of the chain from the inside to the outside of the corona, the end-point distribution has just a single maximum and smoothly goes to zero for large $r$ values and approaches a value of $1 /\left(4 \pi R^{2}\right)$ at the core surface. This result is true for an ideal TM, which is small (similar to an A segment) and for the case that the water has the same solvent quality for the TM as the tether A. We are interested in parameters that deviate from this ideal situation and then there are discontinuities, meaning that there is a minor jumplike 

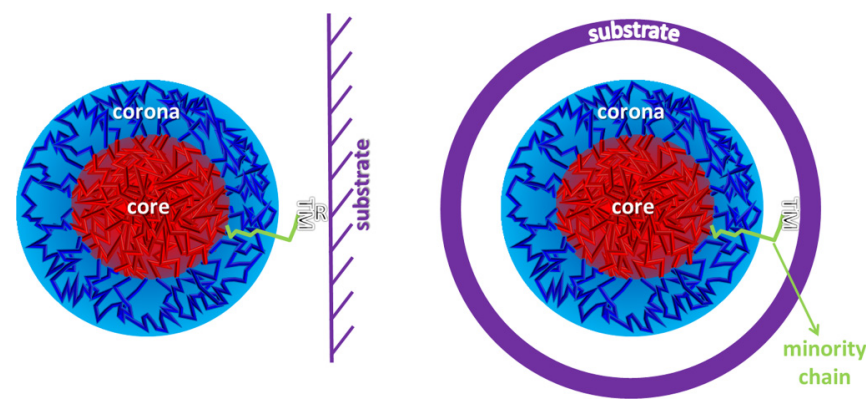

FIG. 3. Schematic two-dimensional representation of (left panel) a micelle with a minority chain adsorbing with a targeting moiety (TM) to a receptor (R) site on an external planar substrate (e.g., a cell wall with receptor). Right panel: a central micelle surrounded by a spherical "substrate" as considered in the cell model; the TM of the minority chain adsorbed on this substrate, which is expected to be covered by receptors (not indicated). The core of the micelle is red, the corona blue [a few corona chains (loops) are indicated]. The minority chain is green and has a TM (represented by TM).

transition from the TM being inside to being outside the corona upon, e.g., an increase of the length of the minority chain.

Continuing with the ideal TM case, let us now consider the presence of a surface (receptor) at the upper boundary of the spherical coordinate system, that is, at $r=M+1$. Below, we refer to the position of the receptor by the distance $\Delta r \equiv\left(M-R_{h}\right)$, wherein $M-R_{h}$ is the distance of the receptor from the hydrodynamic radius of the micelle in lattice units. Obviously, as long as an adsorbing receptor surface is present for distances larger than the chain length $\left(M-R>N_{\mathrm{B}}\right)$, the probe chain cannot reach this receptor surface and the end-point distribution is unaffected. However, when the receptor is in close proximity, the end of the probe can reach the receptor. When the end has a significant affinity for the receptor, we expect that the end segment, that is, the targeting moiety (TM), is able to reach and adsorb onto the receptors. By doing so, the probability for the TM to be near the surface will dramatically increase as compared to the value at the same coordinate in the absence of the substrate. A cartoon of this situation is given in Fig. 3(b), which mimics the situation depicted in Fig. 3(a). In this figure, the end point sits at the substrate. In reality, the TM is only located at the receptor surface with a given probability smaller than unity. Insights into binding of end grafted polymers to flat surfaces were obtained by performing computer simulations [37,38]. This case was also studied theoretically [39]. Our interest is in the probability distribution of the end point (receptor), which in fact is proportional to the radial volume fraction profile. The end-point distribution may potentially have two maxima, namely, one in or around the corona (we will call this the central maximum) and one next to the adsorbing surface (we call this the distal maximum) and two minima, namely, between the core and the periphery of the corona (the proximal minimum), and one between the adsorbing surface and the periphery of the corona (the distal minimum).

\section{B. Landau free energy and a short case study}

The free energy of the system $F$ in units of $k_{B} T$ is found by $F=-\ln Q$, where $Q$ is the canonical partition function. In a mean field theory, we can decompose this partition function into so-called single molecule subpartition functions $q_{i}$,

$$
Q=\pi_{i} \frac{q_{i}^{n_{i}}}{n_{i} !},
$$

where $n_{i}$ is the number of molecules of type $i$. Following the line of arguments, it is natural to take a closer look at the minority chain, while assuming that the other subpartition functions are invariant upon changes in the conformational properties of the minority chain, e.g., when it binds onto a nearby receptor. Of course, for this to be true we should insist on the case that the receptor surface remains outside the corona of the micelle in all cases.

The subpartition function for the minority chain in principle should contain the statistical weights of all possible and allowed conformations. The evaluation of this partition function is simplified because the first segment is strictly positioned to be next to the core surface. In practice, the propagator formalism is used here to generate the partition function. In this formalism, there are distribution functions $G(r, s \mid R+1,1)$ that specify the statistical weight of having segment $s$ at coordinate $r$, under the constraint that the first segment $s=1$ is at coordinate $r=R+1$, that is, next to the core. In the propagator equation, this distribution function is computed starting with $s=1$, and ending with segment $s=N$ giving $G(r, N \mid R+1,1)$. Interestingly, this end-point distribution function can be used to directly compute the volume fraction profile of the free end, that is, $\varphi(r, N) \propto$ $G(r, N \mid R+1,1)$. The proportionality constant is $1 / q$, wherein the single chain partition function $q$ is found by summing this quantity over all available coordinates $r$ :

$$
q=\sum_{r} L(r) G(r, N \mid R+1,1) .
$$

Inspired by this relation, we can identify $G\left(r^{\prime}, N \mid R+1,1\right)$ as a positional subpartition function of the probe chain, which starts (per definition) with segment $s=1$ at $r=R+1$ and ends with segment $s=N$ at coordinate $r=r^{\prime}$. The free energy associated with this positional subpartition function is called the Landau free energy $F_{0}(r)$ [40-42], which in units of $k_{B} T$ is given by

$$
F_{0}(r)=-\ln G(r, N \mid R+1,1)=\ln q-\ln \varphi(r, N) .
$$

The constant $\ln q$ can be absorbed into the Landau free energy. Here and below, we will use $F(r) \equiv F_{0}(r)-\ln q$. Hence, the Landau free energy can directly be computed from the radial volume fraction profile of the end point of the minority chain. In the current context, the position of the free end $r$ is often referred to as the order parameter.

In practice, a targeting moiety (TM) consists of a somewhat larger fragment than an ethylene oxide segment. We can mimic this by taking a minority chain with the following architecture: $\mathrm{A}_{1} \mathrm{~B}_{N_{\mathrm{B}}} \mathrm{TM}_{N_{\mathrm{TM}}}$, with $N_{\mathrm{TM}}>1$. We may subsequently generalize the Landau formalism by relating the Landau energy to the volume fraction distribution of all the TM segments, that is, $F(r)=-\ln \varphi_{\mathrm{TM}}(r)$.

At this stage, it is of interest to discuss an example of how we are going to use this Landau free energy. For this we use the minority (probe) chain $\mathrm{A}_{1} \mathrm{~B}_{25} \mathrm{TM}_{5}$. The segments TM are taken similar to that of $\mathrm{B}$, with the exception of the affinity of the TM 

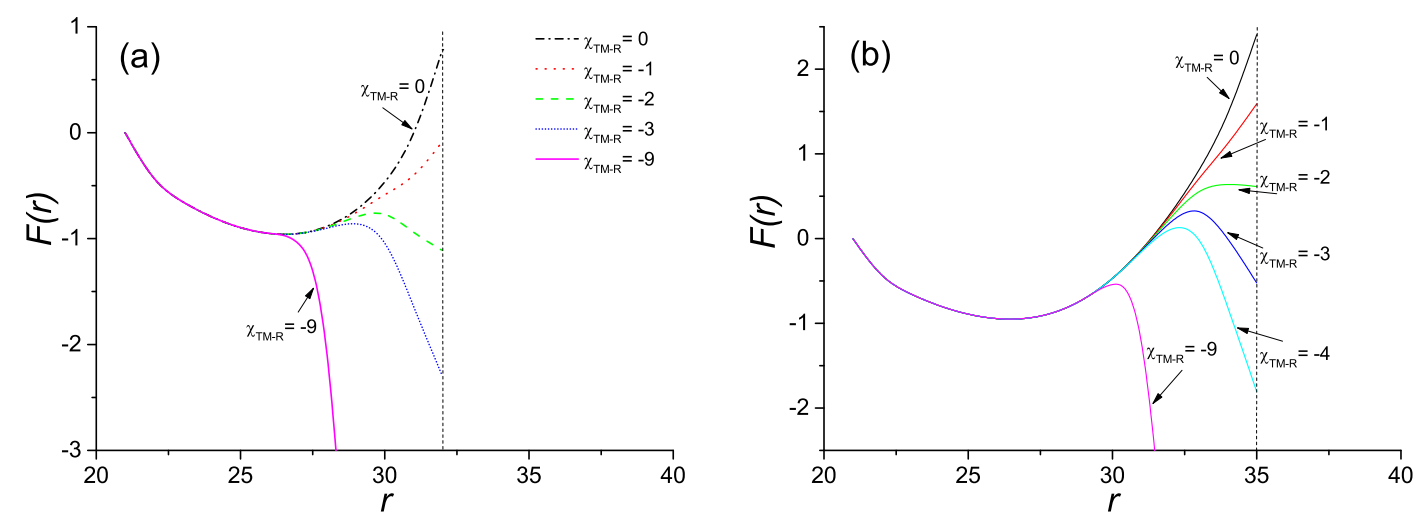

FIG. 4. The dimensionless Landau free energy $F(r)$ (in units of $k_{B} T$ ), where $r$ is the distance to the center of the core for a probe chain $\mathrm{A}_{1} \mathrm{~B}_{25} \mathrm{TM}_{5}$ in lattice site units for various values of the affinity of the TM for the receptor $\chi_{\mathrm{TM}-\mathrm{R}}$ as indicated, for the position of the receptor surface. (a) Position of the receptor surface $r=32$. (b) Position of the receptor surface $r=35$. The Landau free energy is normalized such that $F(16.8)=0$. Note that in panel (a) the curves for $r<27$ overlap. The same happens in panel (b) for $r<30$. The vertical (dashed black) line represents the upper limit of the cell and where the receptor surface is present.

for the receptor surface $\chi_{\mathrm{TM}-\mathrm{R}}$. While all affinities $\chi_{X-R}=0$, the (strong) affinity for the targeting moiety for the receptor is reflected in a negative value for this parameter. Landau free energy curves are presented in Fig. 4. Note that in these computations an excess of multivalent ligands are considered, which is beneficial for targeting. See $[33,34]$ for the case of limited availability of receptors. Without losing generality, one can add a constant to the Landau function. Here, we choose to shift each curve such that all Landau functions attain a zero value at the core side. For illustration purposes, we set the receptor surface very close to the edge of the corona, namely, at $r_{\mathrm{R}}=25.6 \mathrm{~nm}$ in Fig. 4(a) and slightly further outward, that is $r_{\mathrm{R}}=28.0 \mathrm{~nm}$ in Fig. 4(b).

As can be seen in Fig. 4 , as long as $\chi_{\mathrm{TM}-\mathrm{R}}=0$ the Landau function has one central minimum and two maxima. With respect to the core, we have a proximal maximum at $F^{\max }=0$ and a distal maximum at the receptor surface. Of course, this central minimum corresponds to the central maximum in the volume fraction profile (see Fig. 2). With increasing affinity for the receptor a new (distal) minimum develops, that is, near the receptor surface. The distal maximum is naturally moved to lower values of $r$. For a particular value of $\chi_{\mathrm{TM}-\mathrm{R}}^{*}$ the depths of the central and the distal minimum are the same. This occurs for $\chi_{\mathrm{TM}-\mathrm{R}}^{*} \approx-2$ in Fig. 4(a) and $\chi_{\mathrm{TM}-\mathrm{R}}^{*} \approx-3$ for Fig. 4(b). Using state transition terminology, we can identify the equal depth condition as the binodal. The distal maximum in-between the central and distal minimum is identified by the activation barrier that needs to be overcome by the TM to reach the receptor. At the binodal the height of the barrier $U$ does not depend on which minimum is used to measure the height. When $\chi_{\mathrm{TM}-\mathrm{R}}<\chi_{\mathrm{TM}-\mathrm{R}}^{*}$, the height as measured from the central minimum is lower than that measured from the distal minimum. Below, when we discuss the height of the barrier, we will always measure this height taking the central minimum as the reference. The physical origin of the barrier is clear. Before the TM can reach the receptor, it has to stretch even further than its naturally stretched conformation in the corona. This stretching costs entropy and hence the free energy increases. The lowest minimum in $F(r)$ corresponds to the ground state. Taking the affinity for the receptor as the control variable it is clear that at $\chi_{\mathrm{TM}-\mathrm{R}}=\chi_{\mathrm{TM}-\mathrm{R}}^{*}$, the ground state is degenerate. For lower affinities, the lowest free energy is when the TM is inside the brush, whereas at larger affinities the ground state is when the the TM is at the receptor. For successful targeting, the latter is required.

In all cases, the height of the barrier $U$ decreases when $\chi_{\text {TM-R }}$ becomes more negative. In this case, the probability of finding the TM next to the receptor increases more and more and eventually the height $U=0$. This condition is identified by the spinodal of the translocation transition. In Fig. 4(a), this happens for $\chi_{\mathrm{TM}-\mathrm{R}}^{* *} \approx-9$. In Fig. 4(b), the receptor is placed just beyond the corona. In all cases, the height $U$ of the barrier is higher than in Fig. 4(a), and the spinodal condition has shifted to much lower values of $\chi_{\mathrm{TM}-\mathrm{R}}$. When this barrier is gone, all chain ends have moved to the receptor and the "population of conformations" that otherwise would have remained as the unperturbed conformations in the corona has vanished.

Figures 4(a) and 4(b) can be used to understand that $\chi_{\mathrm{TM}-\mathrm{R}}^{*}$ decreases with $r_{\mathrm{R}}$, and that the height of the barrier at the binodal $U^{*}$ increases with $r_{\mathrm{R}}$. Also, the spinodal $\chi_{\mathrm{TM}-\mathrm{R}}^{* *}$ decreases strongly with $r_{\mathrm{R}}$. Clearly, for the minority chain to be a good targeting moiety we need $r_{\mathrm{R}}$ to be larger than the hydrodynamic radius of the micelle, and the affinity for the target should be $\chi_{\mathrm{TM}-\mathrm{R}}<\chi_{\mathrm{TM}-\mathrm{R}}^{*}$. Meanwhile, the barrier $U$ should be not too high. Of course, it is not necessary that the barrier vanishes (spinodal condition) completely. We expect that when $U \approx 3 k_{B} T$ the barrier can easily be crossed and therefore we will monitor aside from the binodal and the spinodal also the $U=3$ situation. Obviously, the latter is only relevant when the barrier at the binodal $U^{*}>3 k_{B} T$.

Before advancing to the results section we need to consider one more complication, namely, when the TM has an affinity for the core. In this case, it is more natural to normalize the Landau free energy so that the value at the receptor surface is zero. In Fig. 5, we show results for the Landau function for which the receptor substrate is set to $r_{\mathrm{R}}=35$, and the TM has, in addition to a mild affinity for the receptor $\chi_{\mathrm{TM}-\mathrm{R}}=-3$ (close to the binodal of the translocation transition), some extra affinity of the TM for the core. A more negative value 


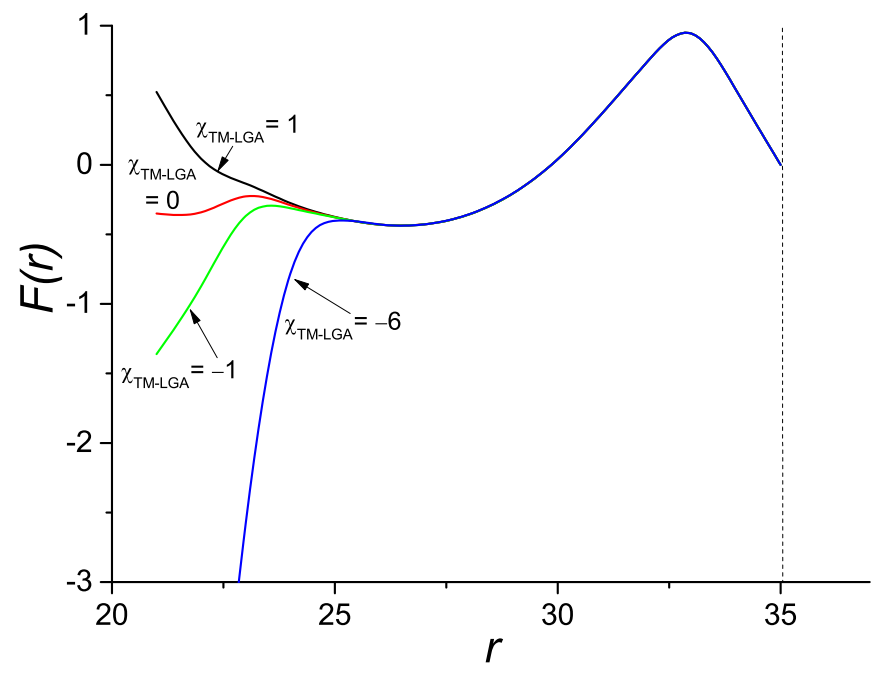

FIG. 5. Landau free energy $F(r)$ (in units of $k_{B} T$ ), $r$ as a function of the distance to the center of the core for a probe chain $\mathrm{A}_{1} \mathrm{~B}_{25} \mathrm{TM}_{5}$ in lattice site units (reminder 1 lattice unit $=0.8 \mathrm{~nm}$ ) for various values of the affinity of the TM for the core (composed of units A) $\chi_{\mathrm{TM}-\mathrm{LGA}}$ as indicated, for the position of the receptor surface (dashed black line) $r_{\mathrm{R}}=35$ and $\chi_{\mathrm{TM}-\mathrm{R}}=-3$. The Landau free energy is normalized such that $F\left(r_{\mathrm{R}}\right)=0$. Note that all curves overlap for $r>25$.

for $\chi_{\mathrm{TM}-\mathrm{LGA}}$ implies stronger adsorption of the TM onto the surface of the core. The curve for $\chi_{\mathrm{TM}-\mathrm{LGA}}=1$ also given in Fig. 4 and is here reproduced for comparison reasons. When $\chi_{\mathrm{TM}-\mathrm{LGA}}<1$, a proximal minimum develops in the Landau free energy and the natural proximal maximum is shifted to a position in-between the proximal minimum and the central minimum. Now, the situation is significantly more complex: there are three minima [the proximal, central, and distal minima (compared to the core)] and two maxima (proximal and distal). Again, we may identify the condition that the proximal and central minima are equally deep. This binodal corresponds to the detachment transition of the TM from the surface and occurs for $\chi_{\text {TM-LGA }}^{*} \approx 0$. We may also identify a spinodal for the detachment transition. In this case, $\chi_{\mathrm{TM}-\mathrm{LGA}}^{* *} \approx-6$ and the barrier between the proximal and central minimum vanishes. When $\chi_{\mathrm{TM}-\mathrm{LGA}}<\chi_{\mathrm{TM}-\mathrm{LGA}}^{* *}$, the proximal maximum and the central minimum are gone and we are left with two minima, namely, a proximal and a distal one with one maximum in-between. When the three minima are equally deep, that is, when $\chi_{\mathrm{TM}-\mathrm{LGA}}=\chi_{\mathrm{TM}-\mathrm{LGA}}^{*}$ and $\chi_{\mathrm{TM}-\mathrm{R}}=\chi_{\mathrm{TM}-\mathrm{R}}^{*}$, we have so-called triple point conditions as three "phases" (types of conformations of the probe chain) can coexist.

From this result we can already extract our first design rule for successfully targeting a receptor. We should avoid a strong affinity of the TM for the substrate. Indeed, it is not expected that the receptor can successfully fish for the TM when it is strongly attached to the core. Of course, as long as the affinity of the TM for the receptor is much higher than that for the core, we should expect that at a sufficient close proximity of the receptor to the micelle, the TM-R contact is the ground state. However, the energy barrier for this case will always be very high and the translocation transition will be a very slow one. That is why, in the following, we will restrict ourselves to the case that the TM has a small repulsion to the core, which technically is equivalent with the situation that there is no affinity with the core. This does not mean that the TM cannot adsorb onto the core. As explained, also when the TM is not very soluble with water, there may be an induced adsorption energy and corresponding high concentration of the TM next to the core. As a result, the Landau free energy will have a primary minimum and when this minimum is deeper than the central one, this will be used to measure the height of the barrier $U^{*}$ for obvious reasons.

Below, the focus is on finding conditions so that the ground state is the distal minimum, while the central minimum is at a position lower than the hydrodynamic radius of the micelle. Then, the minority chain will try to reach the receptor starting from a "hidden" (for the immune system) position. However, the energy barrier $U$ that needs to be overcome to reach the receptor may well be so large that the translocation of the TM towards the receptor is a rare event. The idea is then to monitor the $U=3 k_{B} T$ condition (as measured from the micelle core or corona). The numerical value is an ad hoc estimate below which the receptor is expected to be found fast enough and above which this is too slow. Results can be collected in socalled state diagrams. Such diagrams collect the binodal and spinodal points when one parameter (e.g., the length of the minority chain) is varied and the transition is triggered by some control parameter (e.g., $r_{\mathrm{R}}$ ). The binodal curve(s) in such state diagram give the important information regarding the translocation and/or detachment transitions. The spinodals give information on where the respective barriers vanish. The $U=3\left(k_{B} T\right)$ curve will be presented for discussion purposes only.

\section{SF-SCF parameters}

Similarly as above, we base our analysis on the model derived from the spherical micelle composed of PLGA $_{60}$ PEO $_{60}$ PLGA $_{60}$ chains (as shown previously by $[19,20])$. Again, the core was replaced by a spherical particle composed of PLGA segments with radius $R=16 \mathrm{~nm}$, decorated by 237 chains with length 60 grafted on both ends to the core. These chains are assumed to be composed of PEO and this was modeled by the solvent quality parameter $\chi_{\text {EO- } \mathrm{H}_{2} \mathrm{O}}=0.4$. We used minority chains with lengths $N_{B}=5$, $10,15,20,25,30,35,40,60$, and 120, composed of EO units that are end grafted onto the solid particle, while they have a targeting moiety at their free ends. There are three types of interaction parameters connected to the TM: (i) the affinity of the TM for the core. As argued above, we will avoid large affinities of the TM for the core and here take $\chi_{\mathrm{TM}-\mathrm{LGA}}=1.0$ (recall $\chi_{\mathrm{LGA}-\mathrm{H}_{2} \mathrm{O}}=1.6$ ), (ii) that for the receptor $\chi_{\mathrm{TM}-\mathrm{R}}=-3,-6,-12$, and $-18\left(\right.$ recall $\left.\chi_{\mathrm{R}-\mathrm{H}_{2} \mathrm{O}}=0\right)$ and (iii) the solvent quality $\chi_{\mathrm{TM}-\mathrm{H}_{2} \mathrm{O}}=0.1,0.4,1.0$, and 3.0. On top of this, the length of the TM (the volume of the TM or the repeating TM units) was varied $N_{\mathrm{TM}}=1,3,5$, and 10 . Without mentioned otherwise, just one minority chain was used.

\section{RESULTS AND DISCUSSION}

The relevance of several parameters for the translocation transition of the targeting moiety is conveniently presented in 

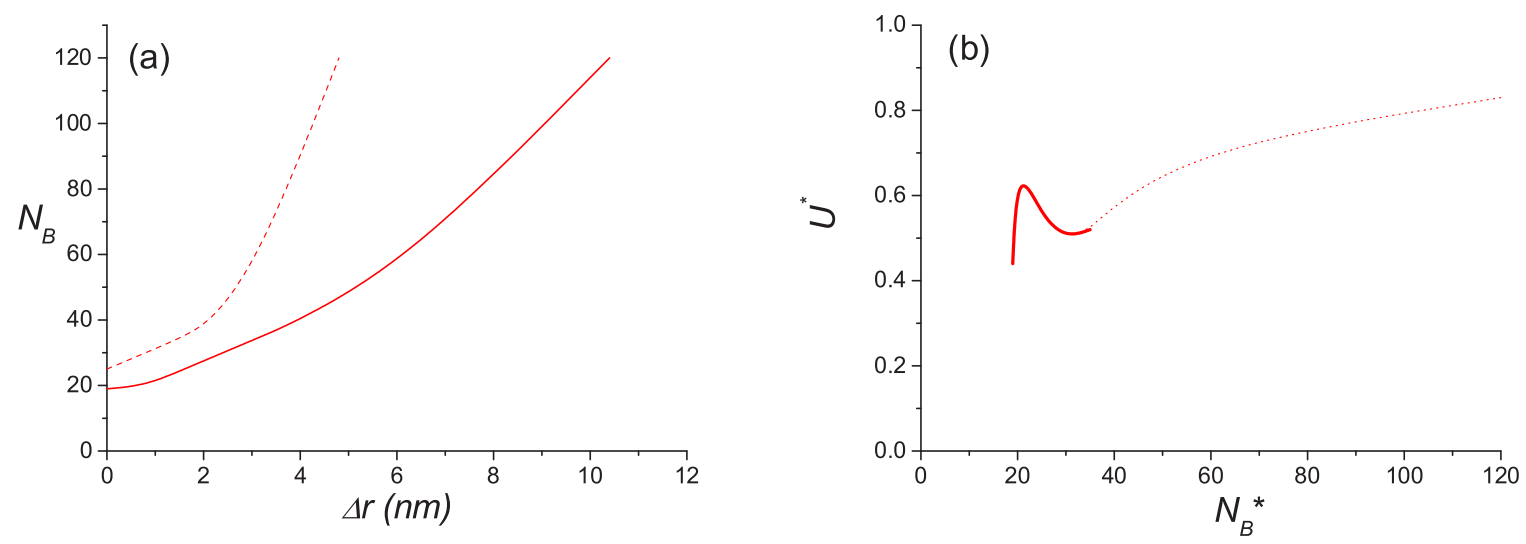

FIG. 6. (a) State diagram in the $\left(N_{\mathrm{B}}, \Delta r\right)$ coordinates. The solid curve is the binodal condition and the dashed curve represents the spinodal. (b) Height of the energy barrier $U^{*}$ (in units of $k_{B} T$ ) at the binodal as a function of the chain length $N_{\mathrm{B}}^{*}$. The solid curve in panel (b) indicates that the TM (in absence of a receptor) is within the hydrodynamic diameter and the dotted curve indicates that the TM is outside the hydrodynamic diameter. Affinity of the TM for the core: $\chi_{\mathrm{TM}-\mathrm{LGA}}=1.0$, for the receptor $\chi_{\mathrm{TM}-\mathrm{R}}=-3$, and $\chi_{\mathrm{TM}-\mathrm{H}_{2} \mathrm{O}}=0.4, N_{\mathrm{TM}}=3$. Other parameters: see Sec. II C.

state diagrams. We will present a representative set of these in the first part of this section. After that, we will zoom in onto the optimized system for the use of polymer micelles as a targeting device for medical (drug delivery) applications.

\section{A. Default state diagram}

Below we adopt the convention to define the position of the receptor surface with respect to the hydrodynamic radius of the micelle, that is, we introduce $\Delta r=r_{\mathrm{R}}-\left(D_{h}\right) / 2$. Hence, $\Delta r=0$ means that the receptor is "touching" the micelle corona layer. Negative values of the receptor distance $\Delta r<0$ (overlap of corona and receptor surface) were disregarded, whereas positive values are of interest (the larger the better). We present state diagrams [cf. Fig. 6(a)] in the coordinates (i) $N_{\mathrm{B}}$, that is the length of the hydrophilic block of the minority chain ( $y$ axis), and (ii) $\Delta r$, which is the distance of the receptor to the micelle surface on the $x$ axis. It is noted that the following conventions are adopted: solid curves represent the binodal condition and dashed curves represent the spinodal. Let us, for completeness, mention how to interpret such state diagram. When for given $N_{\mathrm{B}}$ the TM is far from the micelle, we are to the right of the binodal, and the central minimum is the ground state. Upon reducing $\Delta r$ we approach the binodal curve at $\Delta r=\Delta r^{*}$. At this point, we expect that the TM can thermodynamically reach the receptor $50 \%$ of the time, but this is an activated process as a barrier with height $U^{*}$ needs to be overcome. When the receptor comes even closer to the micelle, the TM-R state is the ground state and the energy barrier is gradually reduced. When the dashed spinodal curve is crossed, at $\Delta r=\Delta r^{* *}$, every attempt of the TM to reach the receptor is expected to be successful as the energy barrier is gone. The height of the energy barrier $U^{*}$ at the binodal as a function of the chain length $N_{\mathrm{B}}^{*}$ at the binodal is additionally presented in panel (b) which accompanies the state diagram, i.e., Fig. 6(b). Here, we have adopted to present the dependence with a solid curve when the TM is sufficiently short, so that in the absence of the receptor the TM is hidden inside the micelle corona. For longer chains, this is no longer the case and part of the chain including the TM is outside the corona (dotted curves).
In this regime, we have used a dashed curve. As long as this energy barrier is sufficiently low, we choose a value of $3 k_{B} T$, the binodal is the most relevant curve in the state diagram, as it demarcates the point from whereon the translocation can effectively take place. However, when this energy barrier is much higher, a point closer to the spinodal is more relevant because of kinetic reasons the binodal is not noticed. At the spinodal translocation occurs spontaneously. This condition is indicated by a dotted curve in the state diagram, corresponding to $U=3 k_{B} T$.

We start with a basic (default) state diagram shown in Fig. 6. It is noted that the chain length $N_{\mathrm{B}}$ of the B block should always be compared to the (hydrophilic) middle block length of the triblock, in this case 60. Here, we have opted for a TM which is 3 segments long and a relatively low value for the affinity for the receptor of $\chi_{\mathrm{TM}-\mathrm{R}}=-3$. Again, we have taken the situation that the minority chain has a vanishing affinity for the core. The current state diagram is representative for all cases that the $\mathrm{TM}$ is repelled by the core or has a very negligible attraction. For a large affinity of the TM for the core the state diagram alters dramatically (not shown). In this case, the energy barrier at the binodal is very small and the curve for $U=3 k_{B} T$ is irrelevant. Inspection of the state diagrams shows that with increasing length of the minority chain, the capture distances (binodal-solid curve) $\Delta r^{*}$ as well as the spinodal conditions $\Delta r^{* *}$ (dashed curves) increases. There exists a minimum value for $N_{\mathrm{B}}$ below which the binodal, and spinodal distances are becoming negative, meaning that the minority chain is too short and the receptor has to penetrate into the micelle before the TM can capture it. The binodal and spinodal curves are not plotted for longer chains than $N_{\mathrm{B}}=120$ either.

For large values of $N_{\mathrm{B}}$ the TM is outside the micelle [see dotted curve in Fig. 6(b)]. In this regime, the minority chains have a flowerlike conformation: they have a strongly stretched stem and a coil-like crown. The crown is outside the micelle and this crown can be used to stretch towards the receptor. For these long chain lengths, the binodal and spinodal distances grow linearly with the length of the minority chain. This indicates that the chain has to stretch proportional to the chain length to reach the receptor. In this limit, the stretching 

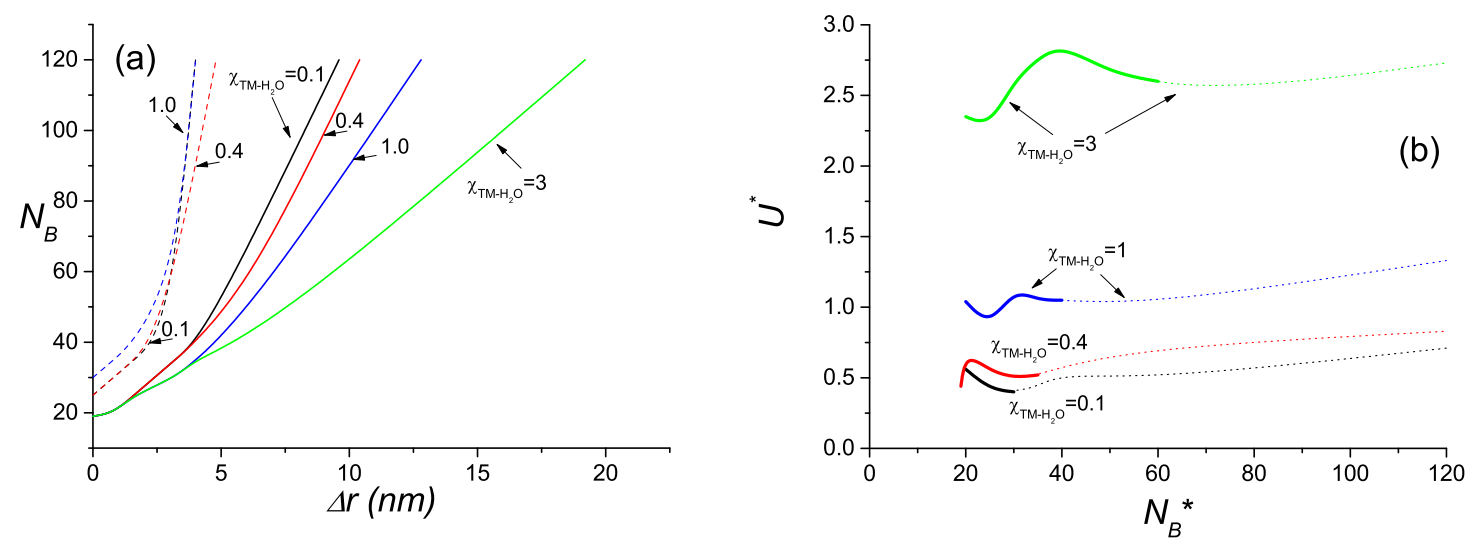

FIG. 7. (a) Collection of state diagrams in the $\left(N_{\mathrm{B}}, \Delta r\right)$ coordinates. The solid curves are the binodal conditions and the dashed curves represent the spinodals. (b) The height of the energy barrier $U^{*}$ at the binodal as a function of the chain length $N_{\mathrm{B}}^{*}$. The solid curves in panel (b) indicate that the TM (in the absence of a receptor) is within the hydrodynamic diameter and the dotted curves indicate that the TM is outside the hydrodynamic diameter. The colors represent different parameters, namely, black: $\chi_{\mathrm{TM}-\mathrm{H}_{2} \mathrm{O}}=0.1$, red: $\chi_{\mathrm{TM}-\mathrm{H}_{2} \mathrm{O}}=0.4$, blue: $\chi_{\mathrm{TM}-\mathrm{H}_{2} \mathrm{O}}=1.0$, and green: $\chi_{\mathrm{TM}-\mathrm{H}_{2} \mathrm{O}}=3.0$ (spinodal curve is for $\Delta r<0$ and therefore not plotted). Other parameters as in Fig. 6.

free energy (conformational entropy loss) is expected to be proportional to the chain length $N_{\mathrm{B}}$ as well and this is in good agreement with the result of Fig. 6(b) for large values of $N_{\mathrm{B}}$. Hence, the barrier $U^{*}$ has an entropic origin. When the TM extends towards the receptor, the chain has a reduced conformational entropy. The position of the barrier is invariably close to the receptor surface because when the TM is at this barrier position, there is already a first contact with the receptor surface (this causes the Landau energy to go down). This means that with increasing length of the TM moiety, the barrier can be increasingly displaced from the receptor surface.

Systems for which $N_{\mathrm{B}}<30$ are most relevant as for these the TM remains inside the corona of the micelle when the receptor is far away. Now, the minority chain is in a more or less homogeneously stretched conformation inside the corona region and the crown is missing. The main difference for shorter $N_{\mathrm{B}}$ is that inside the corona, chains experience a repulsive brush potential that pushes the chain ends outward. This favors the bridging state. As a consequence, the binodal moves out to larger $\Delta r$ values, as can clearly be seen in Fig. 6(a). The binodal has a shoulder at small $N_{\mathrm{B}}$. Since $\Delta r$ is now larger, bridging chains must stretch more. This is probably why the energy barrier increases.

The height of the energy barrier at the binodal [cf. Fig. 6(b)] is very low for small $N_{\mathrm{B}}$. These low values indicate the capture efficiency of the TM for the receptor which is very high already at the binodal. Upon close inspection, a rather complex behavior manifests for $N_{\mathrm{B}}<40$. Apparently, flowerlike conformations $\left(N_{\mathrm{B}}>30\right)$ have a relatively low value for the barrier height at the binodal. Chains that must capture the receptor from inside the corona $\left(N_{\mathrm{B}}<30\right)$ have a relatively high energy barrier: these chains first have to stretch to get out of the corona and then have to stretch further to go to the receptor (adding up to a relatively high $U^{*}$ ). Apparently, as a result, near $N_{\mathrm{B}} \approx 30$, we find $U^{*}$ to drop, but it must be kept in mind that the absolute value of the drop is small (just $\left.0.1 k_{B} T\right)$. Indeed, we can see this nonmonotonous behavior of $U^{*}\left(N_{\mathrm{B}}\right)$ because the absolute values of the barrier heights are small in this case. Below, we find situations that the barrier height is significantly larger and then the irregularities for $U^{*}\left(N_{\mathrm{B}}\right)$ near the value of the length of the minority chain for which flowerlike conformations are found are less visible.

\section{Influence of the solvent quality $\chi_{T M-\mathrm{H}_{2} \mathrm{O}}$}

Obviously, we can construct many different state diagrams and next, in Fig. 7, we will collect four different ones in a so-called collection of state diagrams. The different colors represent different values for the solvent quality of the TM: $\chi_{\mathrm{TM}^{-} \mathrm{H}_{2} \mathrm{O}}(=0.1,0.4,1.0$, and 3.0). Meanwhile, the values of the other parameters in the computations were maintained at $\chi_{\mathrm{TM}-\mathrm{R}}=-3, \chi_{\mathrm{TM}-\mathrm{LGA}}=1.0, N_{\mathrm{TM}}=3$ (as in the default state diagram Fig. 6). With decreasing solvent quality, i.e., increasing $\chi_{\mathrm{TM}-\mathrm{H}_{2} \mathrm{O}}$, the binodal shifts to higher $\Delta r$ values, while the spinodal shifts to lower ones [Fig. 7(a)]. Meanwhile, the height of the energy barrier at the binodal [Fig. 7(b)] increases with decreasing solvent quality. Still, the barrier height remains below the $3 k_{B} T$ value so that the kinetics for the TM-R formation is still expected to remain sufficiently fast. The shift of the binodal with decreasing solvent quality is attributed to the fact that the TM- $R$ affinity also has a solvent quality induced part; the effective adsorption energy increases slightly. Below, we will see that with increasing affinity between the TM and the receptor, the binodal shifts dramatically to the right.

One clear consequence of the increasing repulsion between TM and water is an induced adsorption energy for the TM to the core. This is reflected in progressively longer minority chains that can maintain their TM within the hydrodynamic diameter (in the absence of a receptor), reflected in the length of the solid curves in Fig. 7(b). In more detail, when the TM has an affinity for the hydrophobic core, the minority chain has a looplike conformation, similarly as the corona forming chains. For very poor solvent conditions $\chi_{\mathrm{TM}-\mathrm{H}_{2} \mathrm{O}}=3.0$, the local minimum of the Landau energy near the surface appears and this implies a finite energy barrier near the core (i.e., a barrier associated to the desorption transition) and therefore the spinodal curve suddenly drops to very negative $\Delta r$ values [see Fig. 7(a)]. Fortunately, as the energy barriers still remain 

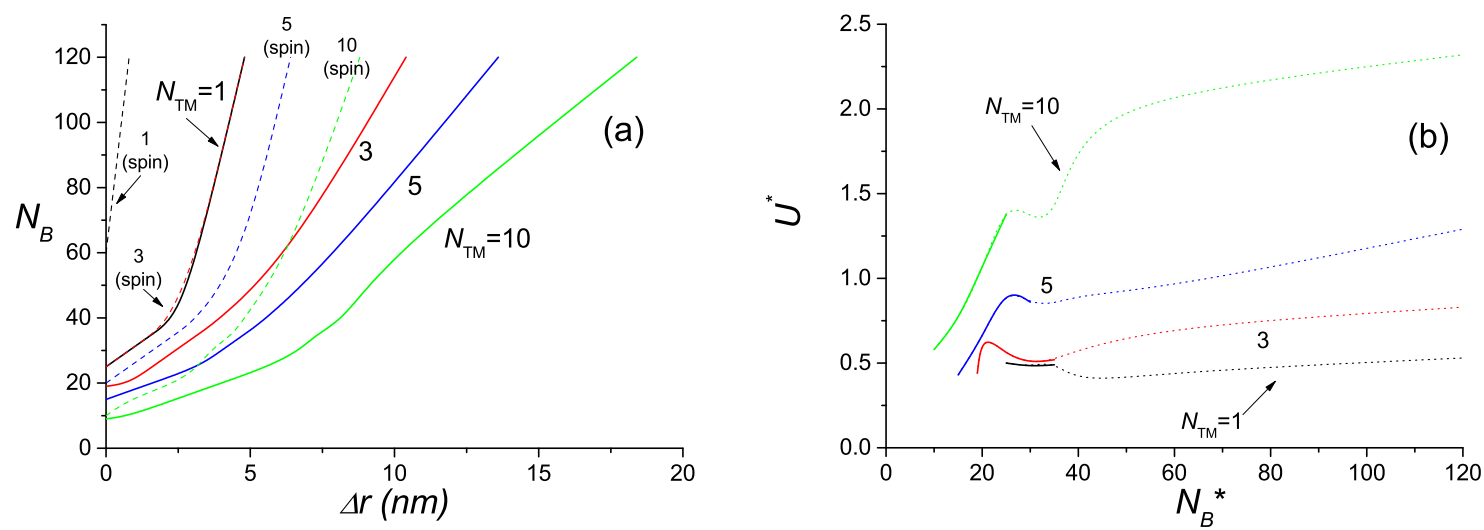

FIG. 8. (a) Various state diagrams in the $N_{\mathrm{B}} \Delta r$ coordinates. The solid curves are the binodal conditions and the dashed curves represent the spinodals. (b) The height of the energy barrier $U^{*}$ at the binodal as a function of the chain length $N_{\mathrm{B}}^{*}$. The solid curves in panel (b) indicate that the TM is within the hydrodynamic diameter (when the receptor is absent) and the dotted curves indicate that the TM is outside the hydrodynamic diameter. The colors represent different lengths of the TM, namely, black: $N_{\mathrm{TM}}=1$, red: $N_{\mathrm{TM}}=3$, blue: $N_{\mathrm{TM}}=5$, and green: $N_{\mathrm{TM}}=10$. Other parameters as in Fig. 6 .

modest, the spinodal curves are not very relevant in this case; the key feature is the binodal.

The intricate behavior found for the $U^{*}\left(N_{\mathrm{B}}\right)$ curves in Fig. 7(b) easily catches our attention. However, the energies involved in these irregularities are very small. On top of the effect discussed above (the flowerlike conformations having a lower barrier than internalized minority chains), we now also have an extra contribution to the desorption transition of the TM from the core. Hence, the extra energy needed to decouple the TM from the hydrophobic core and the stretching (unfolding) of the hydrophilic part of the diblock copolymer may induce one extra maximum and/or minimum in $U^{*}\left(N_{\mathrm{B}}\right)$, noticed for small values of $N_{\mathrm{B}}$.

\section{Influence of $N_{T M}$}

In this section, the attention is drawn to the number of segments at the end of the minority chain. Changes in this value represent a larger or smaller targeting moiety. Alternatively, $N_{\text {TM }}$ can be seen as a series of targeting moieties in a row. As an example, $N_{\mathrm{TM}}=10$ and $\chi_{\mathrm{TM}-\mathrm{R}}=-3$ can be regarded either as a single targeting moiety of $\sim 1 \mathrm{kDa}$ with a total $\chi_{\mathrm{TM}-\mathrm{R}}=-30$ $(10 \times-3)$ or as 10 repetitive targeting moieties each with a $\chi_{\mathrm{TM}-\mathrm{R}}=-3$, the latter interpretation has our preference. The effect of the molar mass or repetition of targeting moiety $\left(N_{\mathrm{TM}}\right)$ units is collected in a collection of state diagrams given in Fig. 8, lengths ranging from 1 to 10 . Other parameters were the same as in the default state diagram Fig. 6.

Inspection of Figs. 8(a) and 8(b) reveals a clear trend that more repeating targeting moieties give a shift of the binodal and spinodal curves to larger $\Delta r$ values and an increase of the corresponding energy barrier at the binodal; again, all energy barriers $\left(U^{*}\right)$ remain below $3 k_{B} T$. These trends may be explained by the fact that more repeating units of the targeting moiety effectively give a stronger TM-R interaction (see also results below). There may also be an entropic effect that as one particular TM-R contact is not realized there is in close vicinity another targeting moiety capable to connect to the receptor. There is a subtle effect that the larger $N_{\text {TM }}$ requires a shorter $N_{\mathrm{B}}$ block lengths to keep the TM within the hydrodynamic diameter see [Fig. 8(b)]. This implies that larger $N_{\mathrm{TM}}$ for the same $N_{\mathrm{B}}$ block lengths establishes TM-R contact at higher $\Delta r$ values. When we add the length of the TM and the spacer $N_{\mathrm{B}}$, we again see that the nonmonotonous behavior of $U^{*}\left(N_{\mathrm{B}}\right)$ occurs at $N_{\mathrm{B}}+N_{\mathrm{TM}} \approx 30$, and thus should be attributed to the (undesired) formation of flowerlike conformations of the minority chain (in the absence of the receptor).

\section{Influence of the affinity of the TM for the receptor $\chi_{T M-R}$}

In this section, we take a closer look to the TM-R affinity parameter. A collection of state diagrams is presented in Fig. 9 for $\chi_{\mathrm{TM}-\mathrm{R}}(-3,-6,-12$, and -18$)$ while the remaining parameters were the same as the default state diagram in Fig. 6. In line with expectations, we observe that the binodal distance shifts strongly, and in a linear fashion, to larger distances with increasingly negative values of $\chi_{\text {TM-R }}$. Remarkably, as can been seen in Fig. 9(a), for this range of parameters the spinodals overlap within the accuracy of the lattice model and hence there is just one dashed red curve. The corresponding values of the energy barrier at the binodal Fig. 9(b) are a linearly increasing function of the absolute value of $\chi_{\mathrm{TM}-\mathrm{R}}$. As the barrier can easily become larger than the threshold value of $3 k_{B} T$ (for the $\chi_{\mathrm{TM}-\mathrm{R}}-3$ and -6 values), we now also present for these cases the $U=3$ curve in Fig. 9(a) (dotted curves). Again, these "practical spinodal" curves are given because we envision that an energy barrier of $3 k_{B} T$ can easily be passed and these practical spinodal values are more relevant than the true spinodal (and the binodal) curves. The practical spinodal distances increase slightly with stronger affinities between TM-R. In passing, we note that with increasing affinity of the TM for the receptor the irregularities in $U^{*}\left(N_{\mathrm{B}}\right)$ still exist but become less clearly visible.

As long as $U^{*}<3$, we take the binodal and above this value the $U=3$ curve to estimate the relevant "capture distance." Inspection of Fig. 9(a) then reveals that with increasing affinity of the TM for the receptor, the capture distance increases continuously, but does not easily go beyond $10 \mathrm{~nm}$ (for $N_{\mathrm{B}}<30$ ) value. 

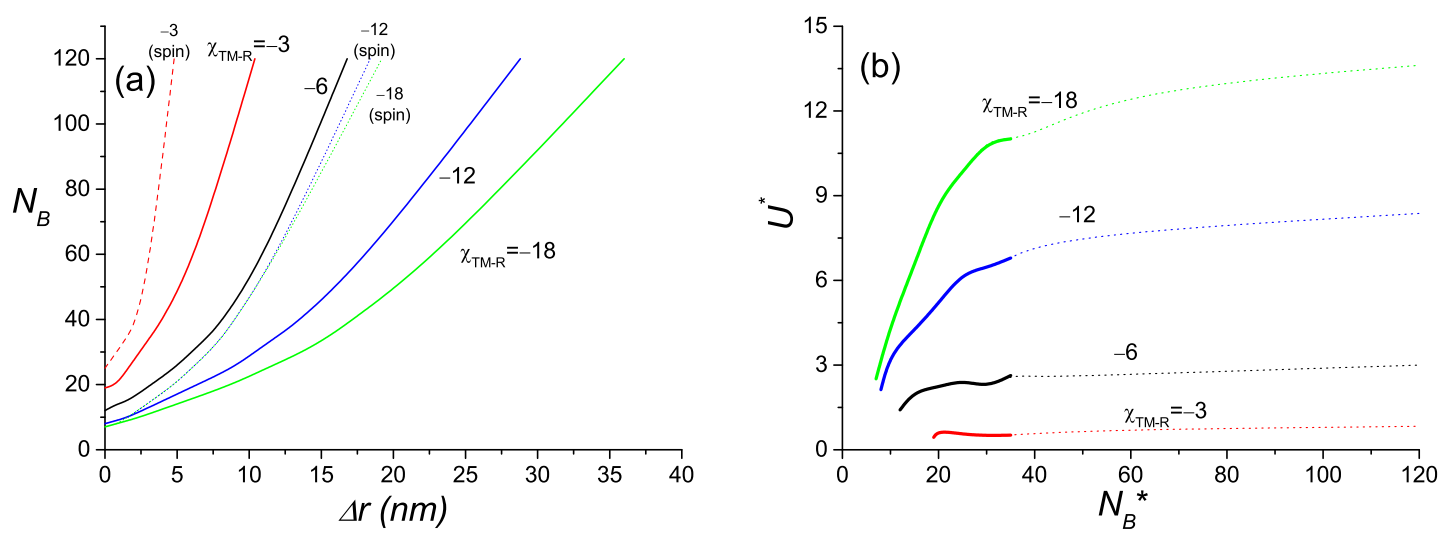

FIG. 9. (a) Collection of state diagrams in the $N_{\mathrm{B}}-\Delta r$ coordinates. The solid curves are the binodal conditions, the dashed curves represent the spinodals, and the dotted curves are drawn for the $U=3 k_{B} T$ condition. (b) The height of the energy barrier $U^{*}$ at the binodal as a function of the chain length $N_{\mathrm{B}}^{*}$. The solid curves in panel (b) indicate that the TM is (when the receptor is absent) within the hydrodynamic diameter and the dotted curves indicate that the TM is outside the hydrodynamic diameter. The colors represent changes in TM-receptor affinity, namely, red: $\chi_{\mathrm{TM}-\mathrm{R}}=-3$, black: $\chi_{\mathrm{TM}-\mathrm{R}}=-6$, blue: $\chi_{\mathrm{TM}-\mathrm{R}}=-12$, and green: $\chi_{\mathrm{TM}-\mathrm{R}}=-18$. Other parameters as in Fig. 6 .

\section{B. Optimization and discussion}

Finally, after the assessment of the relevant modeling input parameters on the state diagram and the free energy barrier $U^{*}$ we now zoom in onto systems with some preferred targeting design. The optimal design obeys to a set of conditions most of which we already encountered above. (i) The TM should not have a strong affinity for the core. As long as the affinity for the receptor exceeds that for the core, i.e., $\chi_{\mathrm{TM}-\mathrm{R}}<\chi_{\mathrm{TM}-\mathrm{LGA}}$, the translocation transition is still expected to occur. But, as soon as the affinity for the core is significant, the total free energy barrier, that is the desorption plus translocation barrier, is expected to be correspondingly higher, so that the kinetics becomes progressively (exponentially) slower. (ii) There exists a minimum affinity of TM for $\mathrm{R}, \chi_{\mathrm{TM}-\mathrm{R}}<-3$. When the affinity is too low, the binding is not expected to take place in a significant fashion. (iii) The TM should not be too hydrophobic, that is, $\chi_{\mathrm{TM}-\mathrm{H}_{2} \mathrm{O}} \lesssim 1$. Various complications are foreseen for hydrophobic TM. First of all, in the preparation the TM may be internalized inside the (glassy) core, and can then only be released when the core is degraded. Second, there is a solvent-induced affinity for the TM to adsorb onto the core. Then, some extra energy is required to desorb the TM from the core. Third, hydrophobic TMs are expected to have nonspecific affinities for apolar substrates (molecules, receptors, tissues, membranes). Binding to these entities will decrease the ultimate efficiency of the targeting micelle. (iv) The minority chain should be short enough so that the TM is kept inside the corona as long as the receptor surface is not in capturing distance. When the minority chain is too short, the translocation is impossible. Hence, the longer the minority chain the better. As long as the TM is ideally repelled by the core, the maximum length of the minority chain is $N_{\mathrm{TM}}+N_{\mathrm{B}} \sim 30$. (v) The number of repeating TMs along the minority chain is preferably not very small. In the current system, we have focused on linear chain architectures. We argue that it stands also as a model for the case that a minority chain has a branched or dendritic end fragment which features several TM to be coupled to one or more receptors, albeit that the details for this will be slightly different. Alternatively, one can include multiple minority chains inside a micelle to enhance the capturing efficiency.

We argue that the capture distance, which is $\Delta r^{*}$ when the energy barrier is low, and the distance $\Delta r$ when $U=3 k_{B} T$, should be as large as possible. When this value is sufficiently large, the minority chain can in practical situations circumvent obstacles and wander around, which is important when the micelle is near the individual receptors (different from our modeling assumption that the receptor is around the micelle). The following results are presented with the idea in mind to maximize the capture distance.

From these requirements we have identified two parameters which deserve a closer investigation, namely, the affinity of the $\mathrm{TM}$ with respect to the receptor $\chi_{\mathrm{TM}-\mathrm{R}}$ (we choose values -3 , $-6,-12$, and -18 ) and the number of repeating units of TM in the minority chain. Larger values of these two parameters give larger capture distances. Other parameters are the same as for the default system (cf. Fig. 6). This means that we still ignore a significant affinity of the TM for the core and insist on good solvent conditions for the $\operatorname{TM}\left(\chi_{\mathrm{TM}-\mathrm{H}_{2} \mathrm{O}}=0.4\right)$. The requirement to focus on the longest possible minority chain prompts us to fix the total length of the minority chain to $N_{\mathrm{TM}}+$ $N_{\mathrm{B}}=35$ (slightly larger than the value advised above to press our luck). In Fig. 10, we present a set of state diagrams in the coordinates $N_{\mathrm{TM}}$ (longer values imply shorter spacer lengths $N_{\mathrm{B}}$ ) versus the receptor distance $\Delta r$. As typically $U^{*}>3$, we only present the spinodal curves (dashed) and the $U=3 k_{B} T$ curves (dotted).

It follows that for $N_{\mathrm{B}}+N_{\mathrm{TM}}=35$, a wide array of spinodals and practical spinodals $U=3 k_{B} T$ can be realized. Let us first discuss the practical spinodals because these are of primary interest for the application. The practical spinodals (dotted curves) for $N_{\mathrm{TM}}=1$ (and $N_{\mathrm{B}}=34$ ) can be realized at $\Delta r=2 \mathrm{~nm}$ for $\chi_{\mathrm{TM}-\mathrm{R}}=-3, \Delta r=4 \mathrm{~nm}$ for $\chi_{\mathrm{TM}-\mathrm{R}}=-6$ and $\Delta r=6 \mathrm{~nm}$ for $\chi_{\mathrm{TM}-\mathrm{R}}=-12$ and -18 (the difference vanishes in the lattice approximations). The larger the $N_{\mathrm{TM}}$ value, the larger $\Delta r$ for the practical spinodals, although for the smaller $\chi_{\text {TM-R }}$ values $(-3$ and -6$)$, the practical spinodals reach a plateau value at $\Delta r \approx 5 \mathrm{~nm}$ for $\chi_{\mathrm{TM}-\mathrm{R}}=-3$ and at 


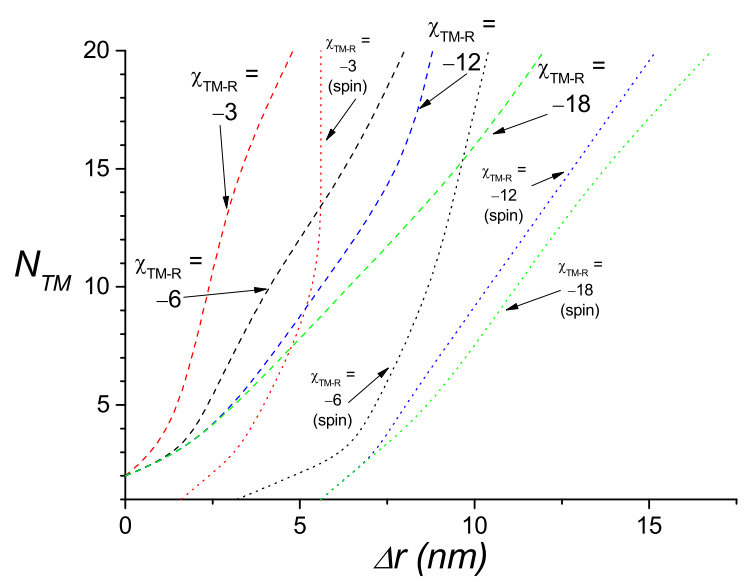

FIG. 10. State diagrams in the $N_{\mathrm{TM}} \Delta r$ coordinates for TM situated within the hydrodynamic diameter. The dotted curves are the practical spinodals $U=3 k_{B} T$ and the dashed curves represent the spinodals. The colors represent different parameters, namely, red: $\chi_{\mathrm{TM}-\mathrm{R}}=-3$, black: $\chi_{\mathrm{TM}-\mathrm{R}}=-6$, blue: $\chi_{\mathrm{TM}-\mathrm{R}}=-12$, and green: $\chi_{\mathrm{TM}-\mathrm{R}}=-18$. The other calculation parameters are similar to the default system (cf. Fig. 6). Here, we have chosen for the longest possible minority chains, that is, we have implemented the constraint $N_{\mathrm{TM}}+N_{\mathrm{B}}=35$.

$\Delta r \approx 10 \mathrm{~nm}$ for $\chi_{\mathrm{TM}-\mathrm{R}}=-6$. Meanwhile, the practical spinodals for $\chi_{\mathrm{TM}-\mathrm{R}}=-12$ and -18 still show a more or less linear growth of $\Delta r$ with $N_{\mathrm{TM}}$, enabling a record TM-R capturing distance $\Delta r \approx 6 \mathrm{~nm}$ up to $\Delta r \approx 15 \mathrm{~nm}$ for $N_{\mathrm{TM}}=20$. For the higher TM-R affinities, the saturation of the "capture distance" is expected to occur for even larger values of $N_{\mathrm{TM}}$. Note that the practical spinodals for $\chi_{\mathrm{TM}-\mathrm{R}}=-12$ and -18 (dotted blue and green curves) are close to each other. For small values of $N_{\mathrm{TM}}$, the practical spinodals for $\chi_{\mathrm{TM}-\mathrm{R}}=-12$ and -18 effectively coincide, while for larger values of $N_{\mathrm{TM}}$ a constant difference of just $\approx 1 \mathrm{~nm}$ for $\Delta r$ is maintained. This implies that the capture distance is not easily increased by increasing the affinity $\chi_{\text {TM-R }}$ to even larger values. However, increasing $N_{\mathrm{TM}}$ remains effective as a means to increase the capture distance as mentioned already. We will elaborate on why this is the case below.

The difference between the thermodynamic spinodal (dashed curves) and the practical spinodals (dotted curve) is indicative for the height of the energy barrier at the binodal, i.e., $U^{*}$. When this difference is very small, the value of $U^{*}$ must be a bit larger than $3 k_{B} T$, whereas when the difference is large, the barrier at the binodal is much larger than $3 k_{B} T$ [see also Figs. 6(a) and 6(b)]. With this information, it is of interest to inspect the whereabouts of the spinodals. The spinodals (dashed curves) coincide at $\Delta r^{* *}=0 \mathrm{~nm}$ for $N_{\mathrm{TM}}=2$ (and $N_{\mathrm{B}}=33$ ) for all $\chi_{\mathrm{TM}-\mathrm{R}}$ values. Within the accuracy of the lattice, the spinodal for $\chi_{\mathrm{TM}-\mathrm{R}}=-6,-12$, and -18 overlap until $N_{\mathrm{TM}}=3$ from this value the spinodal for $\chi_{\mathrm{TM}-\mathrm{R}}=-6$ splits off, while the spinodals for $\chi_{\mathrm{TM}-\mathrm{R}}=-12$ and -18 still overlap until $N_{\mathrm{TM}}=5$. The spinodals (dashed curves) in comparison to the practical spinodals (dotted curves) first seem to go in parallel whereafter they achieve a maximum difference and from thereon they converge back to each other. Again, the latter implies that $U^{*}$ increases with increasing
$N_{\mathrm{TM}}$. For the spinodals and practical spinodals of $\chi_{\mathrm{TM}-\mathrm{R}}=-3$ and -6 this behavior can be completely monitored in Fig. 10 . For the spinodal and practical spinodal of $\chi_{\mathrm{TM}-\mathrm{R}}=-12$, this behavior can be seen until the maximum difference between the two spinodals (see blue curves). The spinodal and practical spinodal for $\chi_{\mathrm{TM}-\mathrm{R}}=-18$ (see green curves) only show the parallel part, and the other features are found for $N_{\mathrm{TM}}>20$ (not shown).

From this optimization study, we conclude that it is possible to increase the capture distance to at least $15 \mathrm{~nm}$. This distance is significant as it is of the same order or a bit larger than the size of the corona. Apparently, it is possible to stretch the minority chain by a factor of more than 2 to reach the receptor surface. Of course, the relatively long series of TM contributes to this remarkable finding. A long sequence of TMs at the end of a relatively short spacer allows the position of the barrier to be significantly displaced from the receptor surface. In a good approximation, this distance scales linearly with $N_{\mathrm{TM}}$ (not shown). The further the barrier is displaced from the receptor, the less the minority chain has to stretch to reach the barrier. That is why the overall barrier height can remain so low while the receptor is relatively far away.

Thus far, we only focused on a single minority diblock copolymer per micelle. We note that while using the nanoprecipitation method, the diblock copolymers are distributed randomly over the micelles and hence it is impossible to achieve exactly one diblock copolymer per micelle. This implies that some micelles certainly will contain more diblock copolymers, while others remain empty. In order to prevent that some micelles contain no diblock copolymers, it is advised to aim at an average of, e.g., $\approx 10$ diblocks per micelle in order to achieve that at least the majority of all micelles contain at least one diblock per micelle. Also, in order to increase the probability on TM-R contact we should incorporate more than one diblock copolymer per micelle. We recall that the corona is composed of over 200 triblock copolymers and therefore having 10 minority chains is still expected to be only a slight perturbation. We have checked that this is indeed the case (not shown). In these cases, we found that each minority chain behaves independently from all the others and therefore we simply can linearly add up the capture efficiency. In other words, the probability for a TM-R contact will increase linearly with the number of minority chains per micelle.

Here, we only focused on repeating TM in a linear chain architecture. Other architecture can still be handled by the SF-SCF machinery. It can be that other geometries (starlike or dendrimerlike) have even a more pronounced effect on TM-R contact and $\Delta r$. These could even be used with larger values for $N_{\mathrm{B}}$ while maintaining the TM within the hydrodynamic diameter and enabling TM-R contact at larger $\Delta r$. To study such modifications becomes appropriate when corresponding experimental systems become available.

There are several ways to check the viability of our SF-SCF modeling approach and we hope such experiments will be performed in the near future. One can, for instance, investigate the strength or possibility of a TM-R connection with atomic force microscopy to generate or validate the parameters needed. Another way of checking the efficiency of the hidden targeting moieties within the micellar corona and their receptor interaction is by exposing the micelles to specific antibodies for 
their targeting moieties. These results should then be compared to those obtained with micelles which do not contain targeting moieties with micelles that contain their targeting moieties beyond (flowerlike conformations) and within the micellar corona (homogeneously stretched conformations). A similar experiment can also be performed with an immune response evoking targeting moiety. Again, when the TM is hidden inside the corona, this should avoid an immune response, while a clear immune response is generated when the (too long) minority chains are in the flowerlike conformation which exposes the TM.

\section{CONCLUSIONS}

Copolymer micelles are promising structures for passive and active drug delivery applications. Experimentally, it is attractive to use the nanoprecipitation method to generate micelles with a well-defined structure and composition. This method can be adopted to include targeting moieties without the need to perform elaborate post-processing of the micelles. In this paper, a modeling approach has been presented aimed at finding the best parameters for such composite micelles to perform as active delivery vehicles. We hope that this modeling study allows for a more efficient design of active drug delivery micelles in medical applications.

We have used the Scheutjens-Fleer self-consistent field (SF-SCF) theory in a spherical coordinate system with concentration gradients in one direction, and implemented a coarse grained model with appropriate parameters that closely predict the micellar structure when triblock copolymers with the PLGA-PEO-PLGA architecture self-assemble through the solvent-precipitation method. We postulated that it is possible to admix a small number of diblocks PLGA-PEO into these micelles without affecting the overall micelle structure. We reported an SF-SCF analysis to reveal which diblock copolymer molar masses or block lengths are beyond or hidden within the corona the micelles. We then focused on the hidden ones: these systems avoid the possible immune response upon their application in the human body. At the free end of the diblock copolymer, a targeting moiety (TM) or a series of TMs are attached and we have positioned a receptor surface around a (slightly simplified) composite micelle in a cell model. A parameter search was executed to find the largest possible capture distance, that is, the distance between the receptor and the micelle at which the TM-R contact is efficiently established. We have introduced a Landau free energy as a function of the distance of the receptor (surface) to the micelle and identified the so-called translocation transition, wherein the TM jumplike goes from the hidden position inside the corona to the receptor which is outside the corona. Here, the ad hoc estimate was taken that the free energy barrier (the minority chain has to stretch to reach the receptor) for the translocation transition should be about $3 k_{B} T$ to estimate the experimentally relevant capture distance. We have shown that it is possible to reach capture distances of at least $15 \mathrm{~nm}$. This length corresponds to the size of the corona and implies that it should be possible to double the stretching of the minority chain in the process of capturing the receptor. On top of this, a number of design rules were formulated. For example, we advised that the TM should not be too hydrophobic. It is also better to prevent specific adsorption of the TM onto the core of the micelle. It is expected that the number of minority chains in the micelle can be larger than unity without adverse effects. More chains imply a higher capture efficiency, but increasing the value too much will perturb the micelle structure in unforeseen ways. We also speculated that a branching of the terminal end of the minority chain can be used to further improve the design.

\section{ACKNOWLEDGMENT}

We thank DSM for supporting this project.
[1] K. S. Soppimath, T. M. Aminabhavi, A. R. Kulkarni, and W. E. Rudzinski, J. Controlled Release 70, 1 (2001).

[2] J. Panyam and V. Labhasetwar, Adv. Drug Deliv. Rev. 55, 329 (2003).

[3] N. Kamaly, Z. Xiao, P. M. Valencia, A. F. Radovc-Moreno, and O. C. Farokhzad, Chem. Soc. Rev. 41, 2971 (2012).

[4] J. C. Wright and D. J. Burgess, Long Acting Injections and Implants (Springer, Berlin, 2012).

[5] R. Langer, Nature (London) 392, 5 (1998).

[6] H. Maeda, G. Y. Bharate, and J. Dauwalla, Eur. J. Pharm. Biopharm. 71, 409 (2009).

[7] S. M. Moghimi, A. C. Hunter, and J. C. Murray, Pharmacol. Rev. 53, 283 (2001).

[8] I. Brigger, C. Dubernet, and P. Couvreur, Adv. Drug Deliv. Rev. 54, 631 (2002).

[9] M. L. Hans and A. M. Lowman, Curr. Opin. Solid State Mater. 6, 319 (2002).

[10] G. Gaucher, M.-H. Dufresne, V. P. Sant, N. Kang, D. Maysinger, and J.-C. Leroux, J. Controlled Release 109, 169 (2005).
[11] J. D. Byrne, T. Betancourt, and L. Brannon-Peppas, Adv. Drug Deliv. Rev. 60, 1615 (2008).

[12] D. Lembo and R. Cavalli, Antiviral Chem. Chemotherapy 21, 53 (2010).

[13] S. Prakash, M. Malhorta, W. Shao, C. Tomaro-Duchewneau, and S. Abbasi, Adv. Drug Deliv. Rev. 63, 1340 (2011).

[14] K. Knop, R. Hoogenboom, D. Fisher, and U. S. Schubert, Angew. Chem. Int. Ed. 49, 6288 (2010).

[15] Some commercially available FDA approved PLGA-based pharmaceutical applications, taken from Ref. [4]: PURASORB $\AA$, EXPANSORB ${ }^{\circledR}$, RESOMER ${ }^{\circledR}$, Absorv ${ }^{\circledR}$; some FDA approved implants and drug delivery systems: Vicryl ${ }^{\circledR}$ degradable PLGA suture, Decapeptyl ${ }^{\circledR}$ and Lupron ${ }^{\circledR}$ Depot both PLGA microparticles for the treatment of prostate cancer, Zoladex ${ }^{\circledR}$ a PLGA based implant for the treatment of prostate cancer, breast cancer, and endometriosis, Risperdal ${ }^{\circledR}$ and Consta ${ }^{\circledR}$ PLGA microparticles for antipsychotic drug delivery and Eligard $\AA$ PLGA based degradable drug depot system.

[16] J. M. H. M. Scheutjens and G. J. Fleer, J. Phys. Chem. 83, 1619 (1979). 
[17] J. M. H. M. Scheutjens and G. J. Fleer, J. Phys. Chem. 84, 178 (1980).

[18] J. M. H. M. Scheutjens, G. J. Fleer, and M. A. Cohen Stuart, Colloids Surf. 21, 285 (1986).

[19] J. G. J. L. Lebouille, R. Tuinier, L. F. W. Vleugels, M. A. Cohen Stuart, and F. A. M. Leermakers, Soft Matter 9, 7515 (2013).

[20] J. G. J. L. Lebouille, L. F. W. Vleugels, A. Dias, F. A. M. Leermakers, M. A. Cohen Stuart, and R. Tuinier, Eur. Phys. J. E 36, 107 (2013).

[21] J. G. J. L. Lebouille, T. Kockelkoren, L. F. W. Vleugels, and R. Tuinier, US Patent No. 0223206 A1 (2011).

[22] Y. Matsumura and H. Maeda, Cancer Res. 46, 6387 (1986).

[23] H. Maeda, J. Wu, T. Sawa, Y. Matsumura, and K. Hori, J. Controlled Release 65, 271 (2000).

[24] H. Maeda and Y. Matsumura, Crit. Rev. Ther. Drug Carrier Syst. 6, 193 (1989).

[25] H. Maeda and J. Fang, Adv. Polym. Sci. 193, 103 (2006).

[26] D. E. Owens and N. A. Peppas, Inter. J. Pharm. 307, 93 (2006).

[27] B. Stella, S. Arpicco, M. T. Peracchia, D. Desmaëlle, J. Hoebeke, M. Renoir, J. D'Angelo, L. Gattel, and P. Couvreur, J. Pharm. Sci. 89, 1452 (2000).

[28] J.-C. Olivier, NeuroRx 2, 108 (2005).

[29] O. C. Farokhzad, J. Cheng, B. A. Teply, I. Sherifi, S. Jon, P. W. Kanthoff, J. P. Richie, and R. Langer, Proc. Natl. Acad. Sci. USA 103, 6315 (2006).
[30] A. Béduneau, P. Saulnier, and J.-P. Benoit, Biomaterials 28, 4947 (2007).

[31] Y. B. Patil, U. S. Toti, A. Khdair, L. Ma, and J. Panyam, Biomaterials 30, 859 (2009).

[32] L. I. Klushin, A. M. Skvortsov, and F. A. M. Leermakers, Phys. Rev. E 66, 036114 (2002).

[33] J. I. Martin, C.-Z. Zhang, and Z.-G. Wang, J. Pol. Sci. B: Pol. Phys. 44, 2621 (2006).

[34] C.-Z. Zhang and Z.-G. Wang, Langmuir 23, 13024 (2007).

[35] G. S. Longo, D. H. Thompson, and I. Szleifer, Langmuir 24, 10324 (2008).

[36] S. Wang and E. E. Dormidontova, Phys. Rev. Lett. 109, 238102 (2012).

[37] S. Wang and E. E. Dormidontova, Biomacromolecules 11, 1785 (2010).

[38] S. Wang and E. E. Dormidontova, Soft Matter 7, 4435 (2011).

[39] R. J. Nap, Y.-Y. Won, and I. Szleifer, Soft Matter 8, 1688 (2012).

[40] F. A. M. Leermakers, J. Van Male, and A. M. Skvortsov, Macromolecules 34, 8294 (2001).

[41] A. M. Skvortsov, L. I. Klushin, J. Van Male, and F. A. M. Leermakers, J. Chem. Phys. 115, 1586 (2001).

[42] A. M. Skvortsov, L. I. Klushin, and F. A. M. Leermakers, Europhys. Lett. 58, 292 (2002). 